\title{
Answer To Test Yourself Question: A 19-year-old male presents with progressive distal lower limb pain
}

\author{
R. Ramanan Rajakulasingam ${ }^{1} \cdot$ Miriam Ficial $^{2} \cdot$ Asif Saifuddin $^{1}$
}

Received: 2 April 2021 / Revised: 12 June 2021 / Accepted: 24 June 2021 / Published online: 12 July 2021

(c) Crown 2021

\section{Test yourself answer}

High-grade osteoblastic osteosarcoma arising from a preexisting low-grade central osteosarcoma

\section{Discussion}

The radiograph (Fig. 1) shows a mixed lytic-sclerotic lesion in the distal tibial metadiaphysis expanding the lateral cortex. MRI (Fig. 2) demonstrates an irregular intramedullary lesion with cortical breach and a small posteromedial extraosseous mass. CT (Fig. 3-below) shows heterogeneous sclerosis laterally with a lytic component medially, suggesting a bi-phasic tumour morphology. CT-guided biopsy of both the lytic and sclerotic components revealed a high-grade osteoblastic osteosarcoma (HG-OS) with murine double minute type 2 (MDM2) gene amplification, likely arising from a pre-existing low-grade central osteosarcoma (LGCOS) (Fig. 4).

LG-COS comprises $\sim 2 \%$ of all osteosarcoma sub-types, usually presenting in the 3 rd decade of life with an equal male to female ratio and a strong predilection for long tubular bones [1]. Several imaging appearances have been

The case presentation can be found at https://doi.org/10.1007/ s00256-021-03854-0

R. Ramanan Rajakulasingam

Ramanan.rajakulasingam1@nhs.net

Miriam Ficial

miriam.ficial@nhs.net

Asif Saifuddin

Asif.saiffudin@nhs.net

1 Department of Radiology, Royal National Orthopaedic Hospital, Brockley Hill, Stanmore HA7 4LP, Middlesex, UK

2 Department of Cellular and Molecular Pathology, Royal National Orthopaedic Hospital, Brockley Hill, Stanmore HA7 4LP, Middlesex, UK attributed to LG-COS, in order of decreasing frequency: lytic with coarse incomplete trabeculation, mainly lytic, mainly sclerotic, and a mixed lytic-sclerotic picture [2]. In our case, the low-grade component was seen laterally manifest by heterogeneous medullary sclerosis. The juxtaposition of sclerosis and lysis in LG-COS can mimic fibrous dysplasia (FD), leading to misdiagnosis [3]. An extraosseous mass, cortical breech, and progressive marrow invasion do not however occur in FD. Three distinct histopathological patterns of LG-COS have been reported with some overlap with FD [3]. Crucially, cortical and Haversian system permeation as well as host bone entrapment at the lesion periphery are not seen in FD and MDM2 protein expression can now safely help diagnose LG-COS [3]. Wide local excision is the accepted treatment, but involved surgical margins are associated with a high rate of local recurrence which typically exhibit a higher histological grade with dedifferentiation and potential for metastases [4]. Radiotherapy and chemotherapy are not typically utilised, the latter only in the setting of a recurrent high-grade lesion or possibly a dedifferentiated component at presentation.

We presume that the history of previous fracture is of no relevance, since isolated case reports of OS developing several years after trauma drew no definitive link between the trauma and tumour [5]. Dedifferentiation of LG-COS is rare, estimated at 10-36\% [6] and tends to exhibit a higher histological grade [7]. Some authors regard the high-grade component of a LG-COS as a separate entity, considering it as de-differentiated warranting systemic adjuvant therapy. However, this distinction and treatment approach is not universally accepted, and it is not known whether areas of HG-OS within a LG-COS actually differ from conventional HG-OS regarding local recurrence, metastasis, and overall survival rates.

The demarcation between the bland LG-COS and highgrade component on imaging is consistent with de-differentiation and should warrant further MDM 2 testing. The possibility of a low-grade component due to the aggressive 
Fig. 3 Coronal CT MPR of the right distal tibia showing a heterogeneous sclerotic lesion (thick arrows) in the lateral aspect of the distal tibia with expansion but no destruction of the cortex representing the low-grade central osteosarcoma, and a lytic area (thin arrows) in the centre of the medullary cavity which has permeated the medial tibial cortex representing the dedifferentiated high-grade osteosarcoma

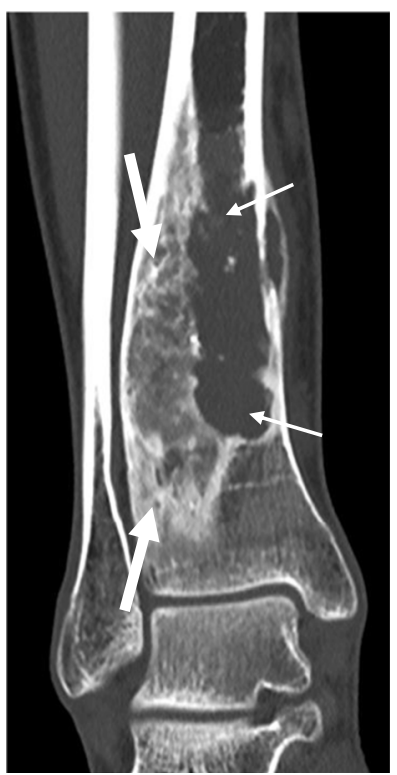

\section{References}

1. Unni KK, Dahlin DC, McLeod RA, Pritchard DJ. Intraosseous well-differentiated osteosarcoma. Cancer. 1977;40(3):1337-47.

2. Andresen KJ, Sundaram M, Unni KK, Sim FH. Imaging features of low-grade central osteosarcoma of the long bones and pelvis. Skeletal Radiol. 2004;33(7):373-9.

3. Bonar SF. Central low-grade osteosarcoma: a diagnostic challenge. Skeletal Radiol. 2012;41(4):365-7.

4. Vasiliadis HS, Arnaoutoglou C, Plakoutsis S, Doukas M, Batistatou A, Xenakis TA. Low-grade central osteosarcoma of distal femur, resembling fibrous dysplasia. World J Orthop. 2013;4(4):327-32.

5. Bennett D, Campbell JR, Brown P. Osteosarcoma associated with healed fractures. J Small Anim Pract. 1979;20(1):13-8.

6. Choi JH, Ro JY. The 2020 WHO classification of tumors of soft tissue: selected changes and new entities. Adv Anat Pathol. 2021;28(1):44-58.

7. Righi A, Paioli A, Dei Tos AP, Gambarotti M, Palmerini E, Cesari M, Marchesi E, Donati DM, Picci P, Ferrari S. High-grade focal areas in low-grade central osteosarcoma: high-grade or still lowgrade osteosarcoma? Clin Sarcoma Res. 2015;29(5):23.

8. Malhas AM, Sumathi VP, James SL, Menna C, Carter SR, Tillman RM, Jeys L, Grimer RJ. Low-grade central osteosarcoma: a difficult condition to diagnose. Sarcoma. 2012;2012:764796.

Publisher's note Springer Nature remains neutral with regard to jurisdictional claims in published maps and institutional affiliations.

\section{Declarations}

Conflict of interest The authors declare no competing interests. 